\title{
Novel Approaches to Individual Alcohol Interventions for Heavy Drinking College Students and Young Adults
}

\author{
Kelly S. DeMartini • Lisa M. Fucito • \\ Stephanie S. O'Malley
}

Published online: 31 January 2015

(C) Springer International Publishing AG 2015

\begin{abstract}
Efficacious alcohol interventions for college students and young adults have been developed but produce small effects of limited duration. This paper provides a review and critique of novel (e.g., a significant deviation from a traditional, brief, and motivational intervention) interventions published between 2009 and 2014 to reduce alcohol use in this population and covers intervention format/components and efficacy on alcohol outcomes. We reviewed 12 randomized controlled trials of novel, individual-level alcohol interventions that reported alcohol outcomes. Four domains of novel interventions are discussed: content (e.g., pharmacotherapy and automatic action tendency retraining), setting (e.g., health centers and ED), modality (e.g., mobile technology), and treatment integration. Findings were mixed for intervention efficacy to reduce amount and frequency of alcohol consumption. Few studies assessed impact on alcohol-related problems. Despite the prevalence of efficacious interventions, there is still an urgent need for novel treatment approaches and delivery mechanisms for this difficult-to-treat population.
\end{abstract}

Keywords Alcohol · College - Intervention · Young adult . Alcohol-related consequences $\cdot$ Technology

This article is part of the Topical Collection on Alcohol

K. S. DeMartini $(\bowtie) \cdot$ L. M. Fucito

Department of Psychiatry, Yale School of Medicine, 1 Long Wharf

Drive, Box 18, New Haven, CT 06511, USA

e-mail: kelly.demartini@yale.edu

L. M. Fucito

e-mail: lisa.fucito@yale.edu

S. S. O'Malley

Department of Psychiatry, Yale School of Medicine and Yale

Comprehensive Cancer Center, Connecticut Mental Health Center -

SAC 202, 34 Park St., New Haven, CT 06519, USA

e-mail: stephanie.omalley@yale.edu

\section{Introduction}

High rates of alcohol consumption and alcohol use disorders in college students and young adults are a substantial public health concern. Heavy episodic drinking, defined as the consumption of five or more drinks on one occasion for a man and the consumption of four or more drinks for a woman, is common; four in ten students engage in heavy drinking $[1 \cdot 2]$. Heavy drinking episodes, in particular, are associated with greater alcohol-related consequences, including physical injury and unprotected sex [3]. While college students are significantly more likely to consume a higher maximum number of drinks, college students and their non-college attending peers report typical average quantities of alcohol consumed that are similar [4]. Moreover, many students and young adults meet diagnostic criteria for an alcohol use disorder. Approximately $32 \%$ of college students meet the criteria for past-year alcohol abuse; approximately $6 \%$ meet the criteria for alcohol dependence [5]. Non-college attending young adults also experience high rates of alcohol use disorders [4], and recent estimates indicate that after adjusting for background sociodemographic characteristics, the risk of any alcohol use disorder is the same for both college students and their non-college attending peers [6]. These persistently high rates of alcohol consumption and associated problems highlight the urgent need for the development of efficacious interventions.

To address this known problem, research on the development of alcohol interventions for this age cohort has burgeoned in the past few decades. Colleges and universities have bolstered efforts to reduce alcohol consumption among their students. Indeed, a recent survey of college administrators from 3514 -year colleges indicated that nearly all (98\%) provide some type of educational program to address alcohol consumption [7]. Only $50 \%$ of the schools, however, provide programs with documented efficacy [7]. Randomized trials of individual alcohol interventions alone number in the hundreds 
$[8,9,10 \bullet, 11,12]$. Overall, these interventions have included mostly first year college student volunteers [10•], and most trials are conducted at large, public universities [8].

Research on these individual alcohol interventions for college students and young adults has produced interventions that effectively reduce rates of alcohol consumption and alcoholrelated problems $[8,13,14]$. Meta-analysis results indicate that the alcohol interventions that are most effective at reducing consumption and problems are individual, face-to-face interventions that utilize motivational interviewing techniques and include personalized, normative alcohol consumption feedback [8]. Accordingly, several of these face-to-face interventions are considered recommended Tier 1 interventions by the National Institute on Alcohol Abuse and Alcoholism [15]. These interventions, however, produce relatively small effects of limited duration and are less effective for the heaviest drinkers or other high-risk groups of young adults or students [8]. Moreover, because this type of intervention requires an inperson intervention session with a counselor, they can be costly, labor-intensive, and hard to disseminate on a large-scale [8].

To address the challenges of face-to-face interventions and meet the needs of college students and young adults, numerous randomized clinical trials have investigated the efficacy of computer-delivered individual brief alcohol interventions. It has been suggested that computer-delivered interventions are well suited to the lifestyle of young adults and that young adults prefer them $[16,17]$. In accordance with the recent increase in interest in these interventions, the efficacy of computer-delivered interventions has been recently reviewed in both narrative reviews and meta-analyses [9, 18]. When compared with wait-list, no-treatment, and assessment-only treatment conditions, the effects of computer-delivered interventions are positive but small. When compared with other active interventions, meta-analysis results indicate that computer-delivered interventions do not show an advantage [9]. A more recent meta-analysis reviewed individual alcohol interventions conducted between 1998 and 2010 and included trials that directly compared computerdelivered interventions with face-to-face interventions [10•]. When these intervention modalities were directly compared, participants reported greater decreases in quantity (including peak blood alcohol concentration) and alcohol-related problems when they received a face-toface intervention [10•]. Therefore, despite the fact that participants who received computer-delivered interventions had better alcohol outcomes than if they received no intervention or a control condition, these interventions have not been able to yield better outcomes than traditional in-person, motivational interventions. Computerdelivered interventions, however, offer significant advantages in terms of clinician-time and cost-efficiency and may have particular utility for early intervention.
It is clear, therefore, that more work needs to be done to build interventions that produce larger effect sizes for young adults in general and/or are more effective at reducing alcohol consumption and alcohol-related problems for heavier and atrisk drinkers. As noted, the majority of individual alcohol interventions, both the face-to-face and computerized, for this population use motivational interviewing techniques, are built around the efficacious Brief Alcohol Screening and Intervention for College Students (BASICS; [19]) model, include personalized feedback, and are conducted with mostly freshmen on the campuses of colleges and universities. Developing and testing interventions that significantly differ from this model could be one way to improve both alcohol consumption and problems outcomes for young adult drinkers.

The purpose of this review is twofold. The first aim is to provide an overview of treatment components in novel, individual-level alcohol interventions (both face-to-face and via other modalities, including technologically-based interventions) designed to reduce alcohol consumption in young adults. The second aim is to summarize the efficacy of these interventions to reduce alcohol consumption outcomes. To be included, studies needed to (a) be specifically designed to reduce alcohol use in college students and/or young adults; (b) use random assignment to treatment conditions; and (c) report outcomes related to alcohol consumption. Importantly, to be included, studies had to be considered novel. As will be further detailed below, interventions were considered novel if they represented a significant deviation from a traditional, brief, and motivational intervention (either computerized or face-to-face). The deviation had to be such that the efficacy of the intervention could be impacted by the deviation. Studies were excluded if they evaluated interventions that were not administered to individuals (i.e., were administered campuswide), because the intervention dose could not be determined in these trials.

\section{Method}

\section{Sample of Studies}

Several search strategies were used to obtain relevant published or in press manuscripts. First, we conducted database searches of PsycINFO, PsycARTICLES, and PubMed using a Boolean search strategy with the following key words: alcohol and (college or university) and intervention. Additional searches were conducted with the following key words: alcohol and young adults and (intervention or treatment). Second, we reviewed the reference sections of relevant published manuscripts. Third, we sent requests for in press manuscripts to the authors. 


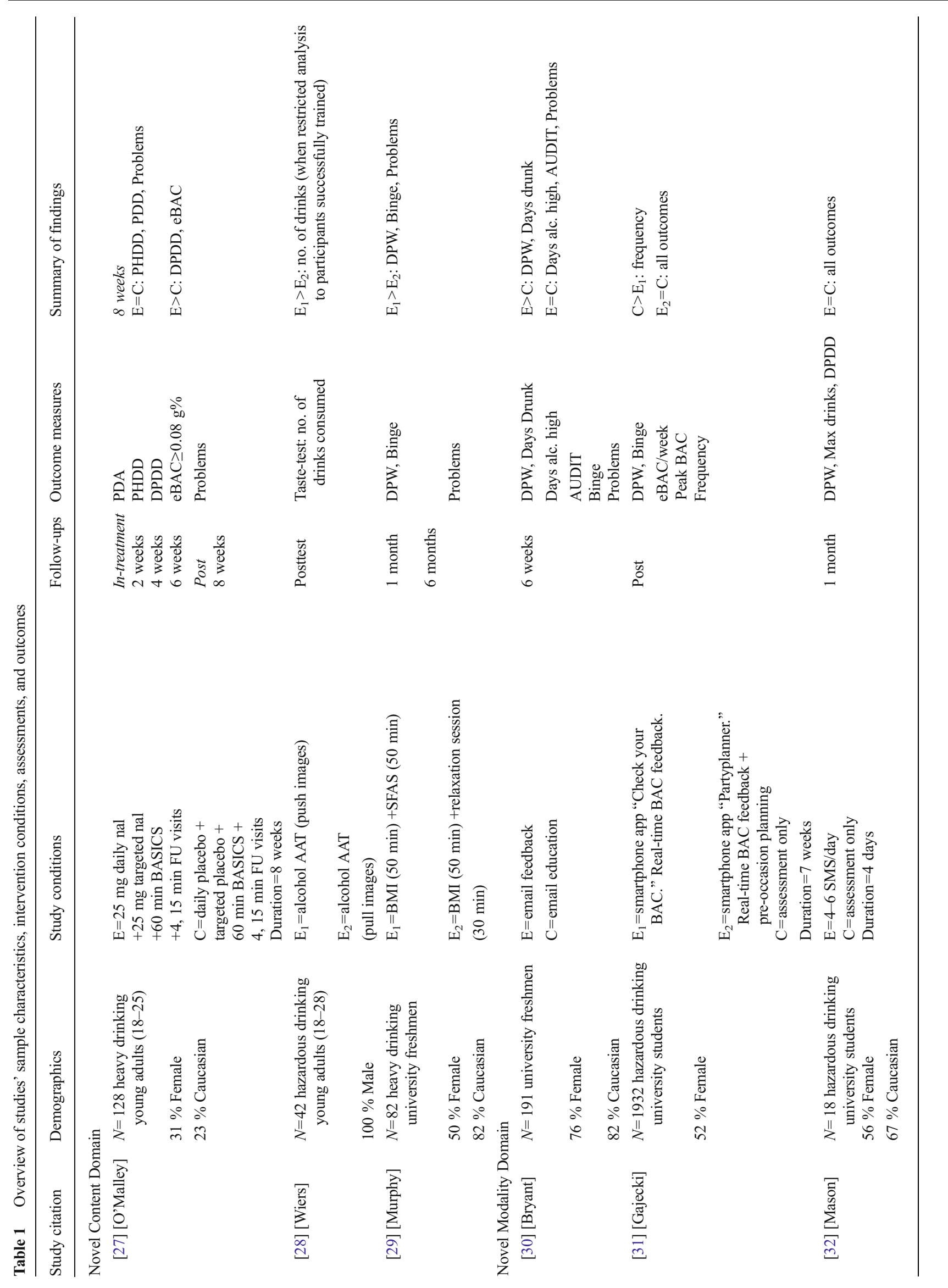




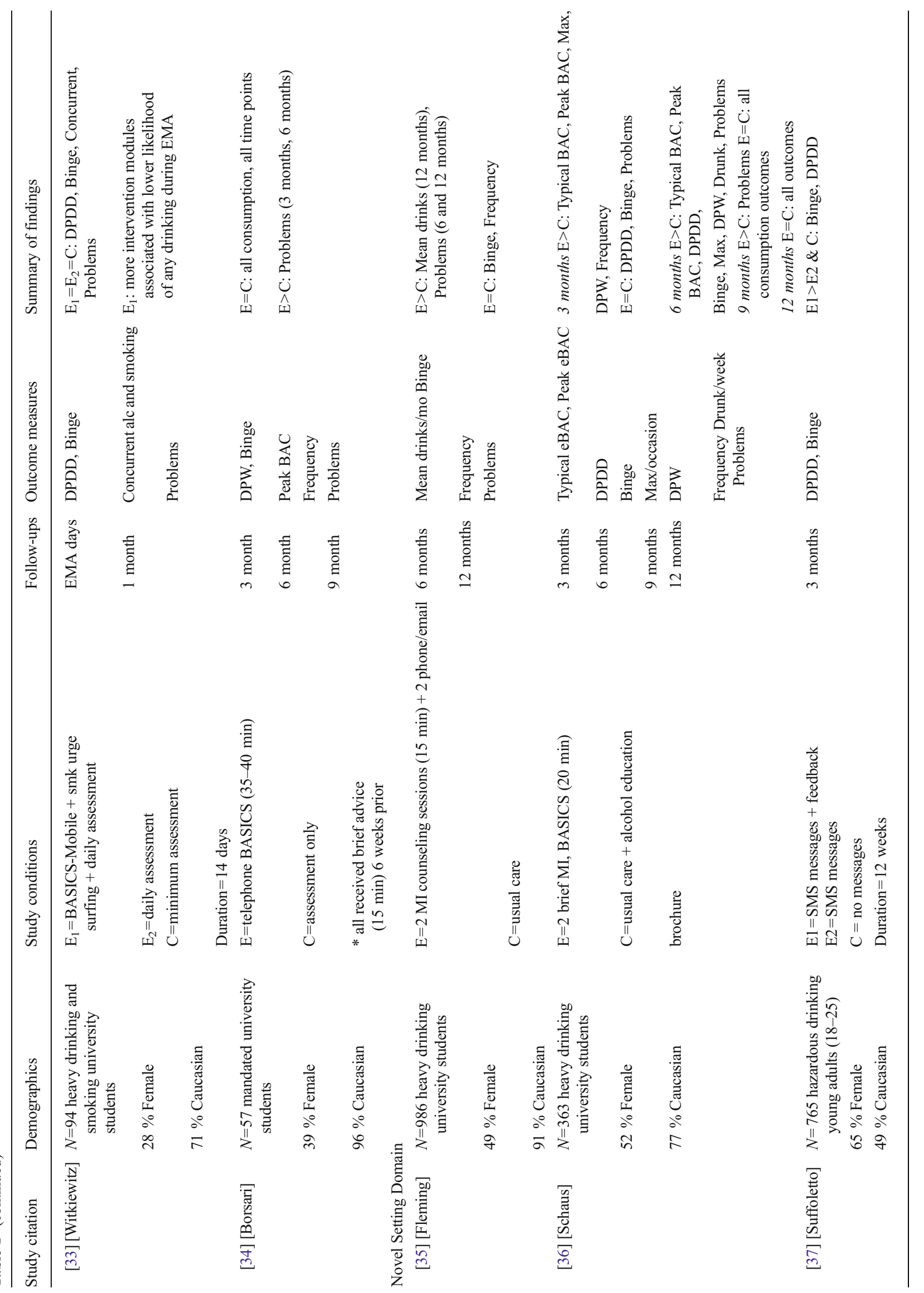




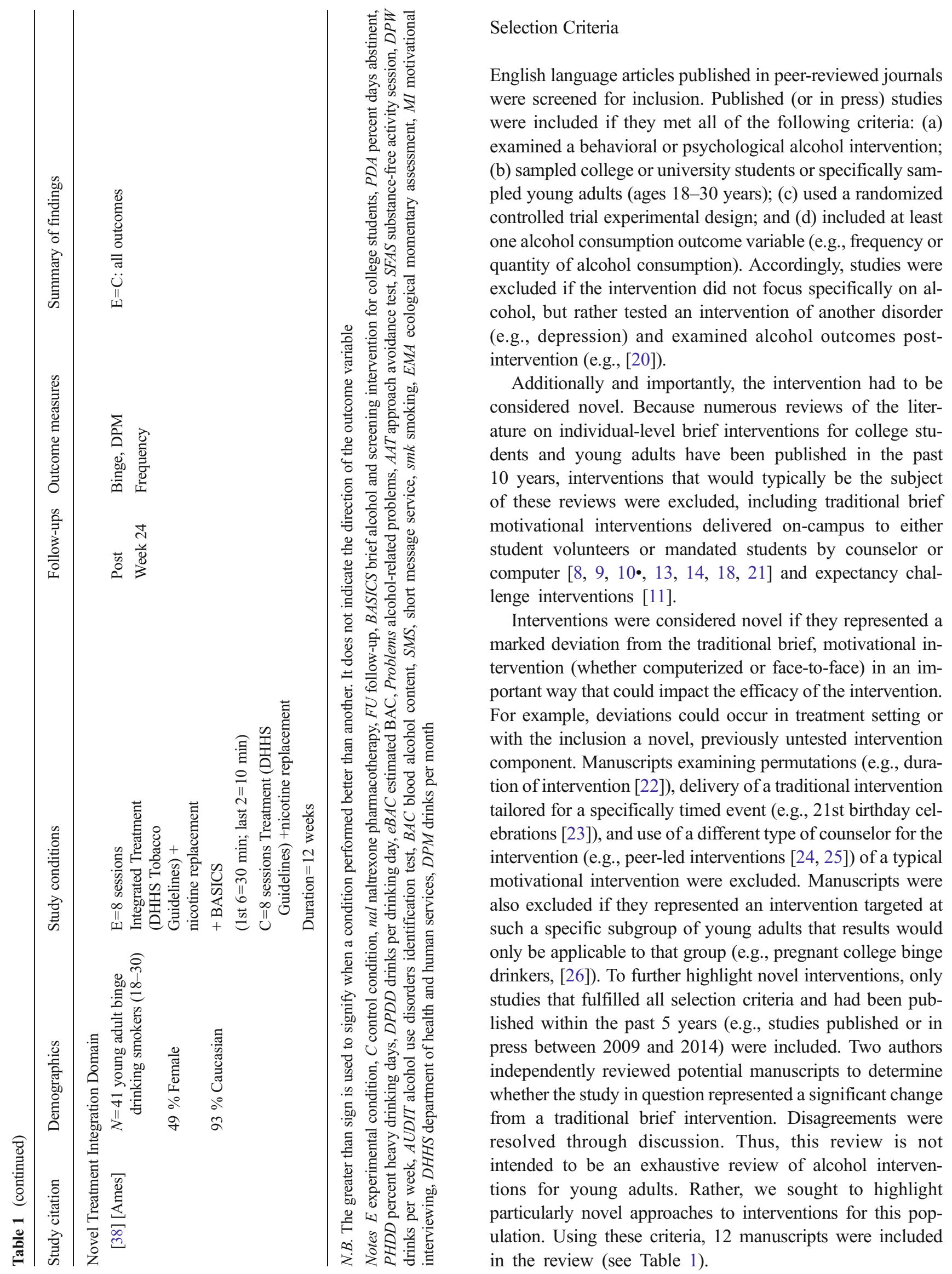




\section{Results}

Between 2009 and 2014, 12 novel randomized controlled trials of alcohol interventions for college students and young adults had been published or were in press. The studies evaluated a wide range of interventions of varying modalities across a number of different treatment settings. Intervention format also varied widely from brief feedback to text message to pharmacological treatment. To provide an organizational framework for review, studies were clustered by the domain on which they were considered most novel. Four novel domains were represented: (a) content $(k=3)$, (b) modality ( $k=$ 5), (c) setting $(k=3)$, and (d) treatment integration $(k=1)$. It should be noted that some studies could have been considered novel in multiple domains. In those cases, we elected to categorize them where we considered them to be most novel and note which studies could have differently categorized. The categories, therefore, serve as a heuristic guide for the reader. Novel content interventions were interventions that contained components that have not been typically included in a brief, motivational interviewing-based, face-to-face, or computerized intervention. Treatment components could be added onto a BASICS intervention (e.g., pharmacotherapy and a behavioral economics module) or could be a totally unique intervention (e.g., cognitive retraining). Studies with novel treatment modalities were studies in which the method of intervention delivery was different from either a counselor-based, face-toface intervention, or a computerized program. This included text-message-based and smartphone application. Lastly, the trial examining integrated treatment was a trial that integrated treatment for binge drinking into an evidence-based treatment for smoking cessation. Taken together, these trials each represented a marked deviation from a traditional face-to-face or computerized brief motivational intervention.

Within each domain, an overview of the intervention approach(es) and study design(s) is reviewed; this is followed by a summary of intervention efficacy on alcohol outcomes. This organizational approach was selected to best highlight the variety of advances in intervention development.

\section{Novel Intervention Content}

Description of Studies Table 1 provides sample characteristics, intervention details, and trial outcomes for the 12 studies reviewed. Three interventions were identified that utilized novel intervention components [27-29]. Of those, two examined the addition of a novel treatment to a traditional, brief motivational intervention [27, 29], and one was a laboratorybased study that examined the impact of a cognitive retraining task [28]. These studies differed dramatically in their content. Focusing first on the two studies that added components to a typical BASICS intervention, one tested pharmacological treatment with naltrexone [27], and the other randomized participants to either BASICS plus relaxation training or BASICS plus a behavioral economic supplement (i.e., SubstanceFree Activity Session, a 50 min individual counseling session that emphasized the salience of students' academic and career goals to highlight the discordance between heavy alcohol consumption and goal achievement [29]). Duration of treatment varied significantly. Pharmacotherapy was administered for 8 weeks and was combined with bi-weekly, 15-20 min sessions with a nurse practitioner who reviewed treatment goals [27]. The behavioral economic supplement was administered in a $50 \mathrm{~min}$, individual counseling session [29]. In both trials, all participants received a BASICS-type individual, motivational intervention approximately $60 \mathrm{~min}$ in length. Follow-up treatment occurred either with a nurse [27] or with relaxation training [29]. The studies had comparable sample sizes of 128 treatment starters [27] and 82 starters [29]. The pharmacotherapy trial included participants between the ages of 18-25 years who reported $\geq 4$ heavy drinking days (e.g., consuming at least four drinks on one drinking occasion for a woman; five or more for a man) in the past month and did not require college attendance. The behavioral economics trial included only college freshmen [27].

The other study in this domain did not test a motivational intervention. The study was a laboratory-based test of a cognitive retraining intervention that examined two variations of the alcohol Approach-Avoidance Task [28]. The study assessed whether the tendency to approach alcohol cues in the environment on the part of heavy drinkers could be changed following a retraining designed to increase or decrease this automatic tendency. The study employed a single-session design in which participants completed a computerized joystick task that asked them to push or pull on the joystick in response to pictures of alcoholic and non-alcoholic beverages. Participants were randomly assigned to either push away (active condition) or pull (control condition) alcohol images toward them. Participants were 42 heavy drinking males (i.e., males that scored at least an 8 on the Alcohol Use Disorders Identification Test (AUDIT; Babor et al., 2001) between the ages of 18-28 years [28]).

Intervention Efficacy On alcohol consumption outcomes, both the pharmacotherapy trial and the cognitive retraining study had significant effects on quantity. At the end of 8 weeks of treatment, pharmacotherapy reduced typical drinks consumed per drinking day [27]. Examination of the whole sample indicated that cognitive retraining had no effect on quantity consumed after the trials. When data from only those who were successfully trained (e.g., those in the "approach" condition who pulled alcohol images toward them more quickly following retraining and those in the "avoid" condition who pushed alcohol images away more quickly after retraining) were examined, at immediate posttest, cognitive retraining reduced number of drinks consumed [28]. Pharmacotherapy 
treatment also significantly reduced the number of drinking days with an estimated blood alcohol content $\geq 0.08 \mathrm{~g} \%$. Long-term follow-up results were not reported [27]. The behavioral economic intervention did not produce a benefit over active control on quantity consumed; both the behavioral economic intervention and active control reduced drinks per week and heavy drinking. Participants in both conditions maintained reductions in heavy drinking at 6 months posttreatment, though the behavioral economics condition had a greater effect size [29]. On alcohol-related problems, the behavioral economic intervention produced an effect that was greater than active control. This was maintained 6 months posttreatment [29]. The cognitive retraining trial did not assess impact on alcohol-related problems [28]. Overall, therefore, pharmacotherapy produced the most durable effects on quantity and the behavioral economic intervention produced the most durable effects on alcohol-related consequences.

\section{Novel Intervention Modality}

Description of Studies Five interventions were identified that utilized a novel intervention modality [30-34]. Three trials evaluated the use of mobile technology for intervention delivery, including text messaging and smartphone applications (EMA) [31-33], one tested a telephone-delivered intervention [34], and one tested an electronic-mail-delivered intervention [30]. Sample sizes ranged from 18 [32] to 1932 [31], and all studies used mixed gender samples of college or university students. One trial used a sample of introductory psychology students [30]; all others used samples of heavy/hazardous drinkers, as defined by either a positive screen on the Alcohol Use Disorders Identification Test or frequency of heavy drinking episodes [31-34], or students mandated to receive intervention due to a campus alcohol violation [34].

Across these five trials, control conditions varied. One trial used a no-intervention control condition [31], three trials used minimal assessment/assessment-only control conditions [32-34], and one used e-mail-delivered alcohol education [30]. In four of the five trials, participants in active treatment conditions received interventions based on principles of motivational interviewing [30, 32-34]. Of the studies that utilized mobile technology for active treatment conditions, one tested an MI-based, text-message delivered intervention [32]; and two tested mobile-based applications either with (e.g., daily [33]) or without [31] text-message prompts. Mobile applications included either real-time electronic blood alcohol content with or without the ability to pre-plan a drinking episode [31] or contained BASICS material and feedback [33]. Active treatment duration also varied significantly, ranging from 7 weeks of mobile application access to one email/phone call with personalized alcohol feedback [30, 34]. Follow-up assessments occurred at a range of time-points, including immediate posttreatment [31], 1 month posttreatment $(n=2)$ [32,
33], 6 weeks posttreatment [30], and 3-, 6-, and 9-months posttreatment [34].

Intervention Efficacy Of the five interventions, two reported significant group effects on alcohol consumption [30, 33]. Relative to control, personalized feedback via e-mail reduced number of drinks on a typical day and number of days that participants felt drunk 6 weeks after intervention [30]. Receiving more modules of text message plus mobile application intervention, including daily assessments, was associated with a decreased likelihood of any alcohol consumption on a given occasion during treatment only compared to control condition. This effect was not seen at 1-month follow-up [33]. Phone intervention, text-message-only intervention, and estimated BAC (e-BAC) feedback plus mobile application were not different from control on alcohol consumption outcomes [31, 32, 34]. Estimated BAC feedback only significantly increased the frequency of alcohol consumption [31], and e-mail alcohol education significantly increased typical drinks per drinking day [30]. Effectiveness of intervention on alcohol-related problems was only assessed in one trial. Compared to control, telephone-delivered intervention significantly reduced alcohol-related problems at 3- and 6-month follow-up [34]. Overall, mobile technology interventions produced weak findings on alcohol consumption, and few studies assessed impact on alcohol-related problems.

\section{Novel Intervention Setting}

Description of Studies Three interventions were identified that utilized a novel intervention setting rather than the typical setting (i.e., a research office) used for the administration of brief motivational interventions [35-37]. Please note that one trial could have also been categorized in the modality section, but was highlighted here because the rationale for the technology used in the study was based on the novel setting [37]. Two of the trials took place in university student health centers [35, 36 ] and one took place in an emergency department [37]. All studies used mixed gender samples of heavy or hazardous university students who screened positive for heavy drinking on either formal assessments (e.g., scores of $\geq 3$ for women and $\geq 4$ for men on the AUDIT) [37] or screened positive by recent history of heavy drinking episodes ( $>14$ drinks per week for men/ >11 drinks per week for women; $>$ five drinks more than four times in the previous 28 days; and/or two or more positive responses to the CAGE [35]; having at least one heavy drinking occasion in the previous 2 weeks [36]). Sample sizes ranged from 363 [36] to 986 [35].

The two trials that took place in university health centers examined the impact of in-person brief treatment sessions delivered by physicians, nurse practitioners, and physician assistants $[35,36]$. The trial that occurred in the emergency department (ED) examined the impact of two active text-message 
interventions: text messages plus feedback and text messages only [37]. Two trials used health brochures/booklets as control conditions [35, 36]; one used a no-treatment control [37]. Both in-person interventions were delivered in two 15-20 min treatment sessions [35, 36]; text message interventions were provided for 12 weeks [37]. Additional contact with a health provider by phone or email was provided in only one trial [35]. Posttreatment follow-up assessments occurred at a minimum of 3 months after treatment $[36,37]$ and a maximum of 12 months $[35,37]$.

Intervention Efficacy All three trials reported significant reductions in alcohol consumption compared to control. At 3 months posttreatment, the text message plus feedback intervention for ED patients had significantly reduced binge drinking and drinks consumed on weekends [37]. The brief intervention provided at a university health clinic significantly reduced all alcohol consumption outcomes, including typical drinks per drinking day and typical and peak BAC at 3 months posttreatment. At 6 months, these effects were maintained; at 9 and 12 months, however, there were no differences on quantity consumed [36]. In the third study, there were no differences between brief physician advice and control on drinks per month or heavy drinking episodes at 6 months posttreatment [35]. At 12 months, mean drinks in the past 28 days were significantly less than control. There was no difference on heavy drinking episodes or drinking frequency [35].

Effectiveness of intervention on alcohol-related problems was assessed in two trials [35, 36]. Compared to control, brief physician advice and brief intervention significantly reduced alcohol-related problems at 6 months; brief intervention significantly reduced problems at 9 months; and both treatments significantly reduced problems at 12 months $[35,36]$. Overall, both in-person and text message interventions delivered in these alternative treatment settings were effective at reducing alcohol consumption in heavy drinkers and both in-person treatments demonstrated long-term effectiveness at reducing alcohol-related problems.

\section{Novel Treatment Integration}

Description of Studies One intervention was identified that integrated alcohol treatment with treatment for another condition (i.e., cigarette smoking) [38]. One other study could have also been categorized here, as it also provided integrated treatment for smoking and drinking [33]. Results of that trial are described in the modality section, so as to cluster all technology-based treatments together. In the integrated intervention to be described here, a binge drinking intervention was integrated into treatment for smoking cessation. Participants were 41 young adults (age range: 18-30 years; mean age $=23$ years) who smoked at least ten cigarettes per day for the past 6 months and reported heavy drinking episodes at least twice in the past 3 months. The control condition consisted of eight sessions of individual, semi-structured counseling based on the United States Department of Health and Human Services Clinical Practice Guidelines for Treating Tobacco Use and Dependence plus 8 weeks of nicotine patch therapy. The first six sessions were $30 \mathrm{~min}$ and the last two were $10 \mathrm{~min}$. The intervention condition consisted of eight sessions based on the same U.S. Guidelines plus nicotine replacement therapy but also included BASICS. The sessions were matched for time [38].

Intervention Efficacy Compared to standard treatment, more participants in the integrated condition were abstinent from smoking posttreatment and at 2 months posttreatment although these differences were not statistically significant. At end of treatment and 2 months posttreatment, change from baseline in binge drinking, drinks consumed, and number of drinking days was equivalent between the groups [38].

\section{Conclusions}

The purpose of this review was to provide an overview of novel, individual-level alcohol intervention components, and to summarize the efficacy of these interventions to reduce alcohol consumption and/or alcohol-related problems in college students and young adults. The current narrative review examined 12 randomized clinical trials. These 12 trials were subdivided into four different domains (e.g., components, setting, modality, and treatment integration) that highlight the ways in which new interventions are building upon and extending past models of treatment for college students and young adults. Overall, findings were mixed for reductions in alcohol frequency and alcohol consumption outcomes, and few of these novel treatments examined impact on alcoholrelated problems.

Two domains contained the most interventions that demonstrated efficacy to reduce alcohol consumption and/or alcohol-related problems. The content domain, which included interventions that deviated the most from the traditional $1-$ 2 session brief alcohol intervention (e.g., pharmacotherapy, cognitive retraining, and behavioral economic supplement), contained three interventions that produced change in consumption and/or problems. The setting domain also contained three trials that produced change in consumption and problems; this setting included the trials that contained the longest follow-up assessments and therefore, included the trials that documented the most durable change. This is particularly important because these domains contained trials that demonstrated efficacy and utilized models of treatment (e.g., pharmacotherapy and treatment in health centers/emergency 
departments) that were not limited to mandated students or even solely undergraduate students.

By moving treatment out of the research offices and training physicians and other medical staff to screen for hazardous alcohol use and to deliver brief alcohol interventions, more students and young adults can have access to needed alcohol interventions. This is important because despite high rates of heavy drinking, college students do not often seek out specialty treatment for alcohol problems. Even though college students have a higher risk of experiencing an alcohol use disorder than their non-college attending peers, they are less likely to seek treatment [39]. Rather, many students feel more comfortable seeing primary care providers than therapists [40]. Thus, treatment for college students should not solely rely on self-identification in specialty clinics. Because a higher proportion of students use campus health clinics than campus mental health clinics [41], university health centers have the potential to screen and provide interventions for larger numbers of students. That the trials highlighted in this review reported results indicating durable reduction in alcohol consumption and problems for heavy drinkers provides early evidence for wider dissemination of this treatment model.

The sole pharmacotherapy treatment (i.e., naltrexone) produced intervention effects that were greater than the traditional brief intervention [22]. This is notable because, while single brief interventions are efficacious, their effects often dissipate by 6 months posttreatment, are small, and are weaker for heavy drinkers [8]. Thus, treatments that produce effects greater than these interventions and for at-risk samples are particularly needed. While pharmacotherapy clinical trials are commonplace for treatment of alcohol abuse and dependence in middle aged adults, the focus of this trial on young adults and college students is unique. The results suggest that adjunctive treatment with naltrexone could provide an effective way to treat college students and young adults most in need of intervention services.

As noted in a recent summary of the history of college student alcohol interventions, the past decade has seen a dramatic change in the way in which students and young adults interact with the world. Social network platforms, including Facebook and Twitter, have replaced in-person social communication. Accordingly, the past 5 years have seen a dramatic increase in the number of studied examining text messagebased interventions [42•]. Indeed, more trials reviewed here were text message or mobile-based interventions than any other single modality. Because of their clear dissemination potential, these types of interventions have the theoretical ability to reach more students and young adults than other types of interventions. However, these studies had the most mixed effects. The studies were widely divergent in the number of text messages sent, the duration for which participants received messages, and the content of the messages. Indeed, some of the content, including real-time feedback on blood alcohol level, was shown to increase alcohol consumption [31]. A great deal more basic research on the types of messages in which students are interested, the phrasing of mobile content, the optimal number of messages sent, how and whether these messages should be combined with other intervention modalities, and the optimal duration of time for students to receive messages remains to be done before the potential of these types of interventions is maximized.

Even among these novel interventions, few of them are dramatically divergent from the traditional brief intervention approach. Two trials, cognitive retraining and treatment integration (i.e., incorporating alcohol treatment into the course of treatment for another conditions/disorder) [28, 38], attempt to reach students and young adults in new ways. By integrating alcohol treatment into efficacious smoking treatment, this type of combined intervention could help target drinkers with other conditions that increase their risk of alcohol-related harm [43, 44]. This type of integration could also provide a way for young adults to receive alcohol content if they are more motivated to engage in behavior change for another disorder or condition. Only one integrated treatment that was specifically designed to include an alcohol component was identified; it produced mixed results [38]. Future research could consider this type of approach as a way to engage young adults with additional risk factors. Cognitive retraining provides a particularly novel way to intervene on alcohol use and could be particularly useful for students and young adults with low motivation to change drinking behavior, because it could be perceived as a less threatening intervention. Early results of this approach show promise. Much more work needs to be done to develop truly divergent intervention approaches.

The primary limitation of this review is the narrowness of scope. To create a specific, targeted review and identify novel interventions in an area of literature that has been reviewed numerous times recently, we used narrow search terms to best filter through recent trials. This strategy, while advantageous for reviewing a burgeoning area of science, may have inadvertently eliminated studies that did not include these search terms but were testing new mechanisms for intervention. Future analysis of this area should consider utilizing broader search terms.

This review highlights potential directions for future studies. First, our review found that novel content interventions yielded significant, positive outcomes. Pharmacotherapy, cognitive retraining, and behavioral economics were significant departures from the traditional BASICS model. Researchers should look to other areas of study (e.g., economics and neuroscience) to build new interventions that harness findings from other fields to target alcohol consumption. Second, the findings from the setting section of this review highlight that interventions need to be tested outside of research offices. As young adults and college students do not often seek specialty services for alcohol treatment, more work needs to be done on 
how and where to screen and intervene effectively. Many questions remain unanswered in that domain. With which parts of the health care system do young adults most interact? Are there other co-occurring health problems (e.g., obesity, sleep, anxiety, etc.) that contribute to alcohol consumption for which college students and young adults would be more open to treatment and in which alcohol treatment could be integrated? Finding better ways to engage college students and young adults in alcohol interventions, instead of relying on voluntary research participation or on-campus violations, is an important next step in the alcohol intervention field. Lastly, replication studies of treatment effects are needed. Though some approaches, including cognitive retraining, have been replicated in other populations (see 45, 46), most of these studies are, by our definition, novel ways of conducting interventions and additional confirmatory studies will increase the probability that they will be adopted. Pharmacotherapy with naltrexone is an effective, FDA-approved treatment for alcohol dependence; the findings reviewed here provide further evidence that naltrexone can help reduce sub-clinical levels of heavy drinking. Similarly, brief interventions conducted in emergency departments have been shown to be efficacious in older populations (for review, see 47). The results here suggest that conducting interventions in the ED or similar on-campus locations might be a particularly promising way to reach young adults and college students.

The high rate of heavy alcohol use among college students and non-student young adults continues to be a problem and is associated with a host of negative alcohol-related consequences. Despite the past development of efficacious interventions, more work needs to be done to build interventions that are effective for heavy drinkers and are accessible to more students and young adults. Of the interventions reviewed here, pharmacotherapy and brief interventions delivered in health centers appear to hold particular promise. Overall, however, with limited evidence for consistent and durable change in alcohol consumption and problems in these interventions, an urgent need remains for the development of new treatments for this population.

\section{Compliance with Ethics Guidelines}

Conflict of Interest Dr. O'Malley has served as a consultant to or advisory board member for Pfizer, Alkermes, Arkeo Pharmaceuticals, and the Hazelden Betty Ford Foundation; she is a member of the American Society of Clinical Psychopharmacology/ACNP's Alcohol Clinical Trials Initiative, which has been supported by Abbott Laboratories, Alkermes, Eli Lilly \& Company, Schering Plough, Lundbeck, Janssen, GlaxoSmithKline, Pfizer and Ethypharm; she has received study supplies from Pfizer and contracts from Eli Lilly and NABI Biopharmaceuticals as a study site for a multi-site trial; and is a past partner of Applied Behavioral Research. Drs. DeMartini and Fucito declare no conflicts.

Support for this work was provided by P50 AA012870 (KSD), K05 AA014715 (SSO), and NR014126 (KSD \& LMF) from the National Institute on Alcohol Abuse and Alcoholism, the National Institute of Nursing Research and by the Connecticut Department of Mental Health and Addiction Services. The content is solely the responsibility of the authors and does not necessarily represent the official views of the funding agencies.

Human and Animal Rights and Informed Consent This article includes a review of a study performed by these authors (see O'Malley et al.). All study procedures for that trial were approved by Yale University's Institutional Review Board.

\section{References}

Papers of particular interest, published recently, have been highlighted as:

- Of importance

1. Substance Abuse and Mental Health Services Administration (SAMHSA). Results form the 2010 National Survey on Drug Use and Health: Summary of National Findings (NSDUH Series H-41, HHS Publication No. SMA 11-4658). Rockville, MD: Substance Abuse and Mental Health Services Administration; 2011. This publication provides an epidemiological summary of the current state of college alcohol consumption.

2. Wechsler H, Lee JE, Kuo M, et al. Trends in college binge drinking during a period of increased prevention efforts. Findings from 4 Harvard School of Public Health College Alcohol Student Surveys: 1993-2001. J Am Coll Heal. 2002;51:203-17.

3. Hingson RW, Zha W, Weitzman ER. Magnitude of and trends in alcohol-related mortality and morbidity among U.S. College students ages 18-24, 1998-2005. J Stud Alcohol Drugs Suppl. 2009;16:12-20.

4. Slutske WS. Alcohol use disorders among US college students and their non-college-attending peers. Arch Gen Psychiatry. 2005;62(3):321-7.

5. Knight JR, Wechsler H, Kuo M, et al. Alcohol abuse and dependence among U.S. college students. J Stud Alcohol. 2002;63(3): 263-70.

6. Blanco C, Okuda M, Wright C, Hasin DS, Grant BF, Liu SM, et al. Mental health of college students and their non-college attending peers: Results form the national epidemiologic study on alcohol and related conditions. Arch Gen Psychiatry. 2008;65(12):1429-37.

7. Nelson TF, Toomey TL, Lenk KM, Erickson DJ, Winters KC. Implementation of NIAAA College drinking task force recommendations: How are colleges doing 6 years later? Alcohol Clin Exp Res. 2010;34:1687-93.

8. Carey KB, Scott-Sheldon LA, Carey MP, DeMartini KS. Individual-level interventions to reduce college student drinking: a meta-analytic review. Addict Behav. 2007;32(11):2469-94.

9. Carey KB, Scott-Sheldon LA, Elliott JC, et al. Computer-delivered interventions to reduce college student drinking: a meta-analysis. Addiction. 2009;104(11):1807-19.

10. Carey KB, Scott-Sheldon LA, Elliott JC, et al. Face-to-face versus computer-delivered alcohol interventions for college drinkers: a meta-analytic review, 1998 to 2010. Clin Psychol Rev. 2012;32(8):690-703. Face-to-face versus computer-delivered alcohol interventions for college drinkers: a meta-analytic review, 1998 to 2010.

11. Scott-Sheldon LA, Terry DL, Carey KB, et al. Efficacy of expectancy challenge interventions to reduce college student drinking: a meta-analytic review. Psychol Addict Behav. 2012;26(3):393-405.

12. Scott-Sheldon LA, Carey KB, Elliott JC, et al. Efficacy of alcohol interventions for first-year college students: a meta-analytic review 
of randomized controlled trials. J Consult Clin Psychol. 2014;82(2): 177-88.

13. Larimer ME, Cronce JM. Identification, prevention, and treatment revisited: Individual-focused college drinking prevention strategies. Addict Behav. 2007;32(11):2439-68.

14. Cronce JM, Larimer ME. Individual-focused approaches to the prevention of college student drinking. Alcohol Res Health. 2011;34(2):210-21.

15. National Institute on Alcohol Abuse and Alcoholism N. A call to action: Changing the culture of drinking at U.S. colleges. Final report of the task force on college drinking (NIH Pub. No. 025010). Rockville, MD: U.S. Department of Health and Human Services, National Institutes of Health; 2002.

16. Escoffery C, Miner KR, Adame DD, et al. Internet use for health information among college students. J Am Coll Heal. 2005;53(4): $183-8$.

17. Kypri K, Saunders JB, Gallagher SJ. Acceptability of various brief intervention approaches for hazardous drinking among university students. Alcohol Alcohol. 2003;38(6):626-8.

18. Elliott JC, Carey KB, Bolles JR. Computer-based interventions for college drinking: a qualitative review. Addict Behav. 2008;33(8): 994-1005.

19. Dimeff LA, Baer J, Kivlahan D, Marlatt G. Brief alcohol screening and intervention for college students. New York: The Guilford Press; 1999.

20. Reynolds EK, MacPherson L, Baruch DE, et al. Integration of the brief Behavioral Activation Treatment for Depression (BATD) into a college orientation program: Depression and alcohol outcomes. J Couns Psychol. 2011;58(4):555-64.

21. Larimer ME, Cronce JM, Lee CM, Kilmer JR. Brief intervention in college settings. Alcohol Res Health. 2004;28(2):94-104.

22. Kulesza M, Apperson M, Larimer ME, Copeland AL. Brief alcohol intervention for college drinkers: How brief is? Addict Behav. 2010;35(7):730-3.

23. Neighbors C, Lee CM, Atkins DC, et al. A randomized controlled trial of event-specific prevention strategies for reducing problematic drinking associated with 21st birthday celebrations. J Consult Clin Psychol. 2012;80(5):850-62.

24. Hustad JTP, Mastroleo NR, Kong L, et al. The comparative effectiveness of individual and group brief motivational interventions for mandated college students. Psychol Addict Behav. 2014;28(1):74 84.

25. Mastroleo NR, Oakley WC, Eaton EM, Borsari B. Response of heavy-drinking voluntary and mandated college students to a peer-led brief motivational intervention addressing alcohol use. J Subst Abus Treat. 2014

26. Ceperich SD, Ingersoll KS. Motivational interviewing + feedback intervention to reduce alcohol-exposed pregnancy risk among college binge drinkers: determinants and patterns of response. J Behav Med. 2011;34:381-95.

27. O'Malley SS, Corbin WR, Leeman RF, et al. Naltrexone reduces alcohol drinking in young adults: A double-blind, placebocontrolled clinical trial of efficacy and safety. J Clin Psychiatry. 2014

28. Wiers RW, Rinck M, Kordts R, et al. Retraining automatic actiontendencies to approach alcohol in hazardous drinkers. Addiction. 2010;105:279-87.

29. Murphy JG, Dennhardt AA, Skidmore JR, et al. A randomized controlled trial of a behavioral economic supplement to brief motivational interventions for college drinking. J Consult Clin Psychol. 2012;80(5):876-86.
30. Bryant ZE, Henslee AM, Correia CJ. Testing the effects of e-mailed personalized feedback on risky alcohol use among college students. Addict Behav. 2013;38:2563-7.

31. Gajecki M, Berman AH, Sinadinovic K, et al. Mobile phone brief intervention applications for risky alcohol use among university students: a randomized controlled study. Addict Sci Clin Pract. 2014;9:1-12.

32. Mason M, Benotsch EG, Way T, et al. Text messaging to increase readiness to change alcohol use in college students. J Prim Prev. 2014:35:47-52.

33. Witkiewitz K, Desai SA, Bowen S, et al. Development and Evaluation of a Mobile Intervention for Heavy Drinking and Smoking Among College Students. Psychol Addict Behav. 2014.

34. Borsari B, Short EE, Mastroleo NR, et al. Phone-delivered brief motivational interventions for mandated college students delivered during the summer months. J Subst Abus Treat. 2014;46:592-6.

35. Fleming MF, Balousek SL, Grossberg PM, et al. Brief physician advice for heavy drinking college students: a randomized controlled trial in college health centers. J Stud Alcohol Drugs. 2010;71:2331.

36. Schaus JF, Sole ML, McCoy TP, et al. Alcohol screening and brief intervention in a college student health center: a randomized controlled trial. J Stud Alcohol Drugs Suppl. 2009;16:131-41.

37. Suffoletto B, Kristan J, Callaway C, et al. A text message alcohol intervention for young adult emergency department patients: a randomized clinical trial. Annals of Emergency Medicine. 2014:1-9.

38. Ames SA, Werch CE, Ames GE, et al. Integrated smoking cessation and binge drinking intervention for young adults: a pilot investigation. Ann Behav Med. 2010;40(3):343-9.

39. Blanco C, Okuda M, Wright $\mathrm{C}$, et al. Mental health of college students and their non-college attending peers: Results from the national epidemiological study on alcohol and related conditions. Arch Gen Psychiatry. 2008;65(12):1429-37.

40. American College Health Association (ACHA). Considerations for integration of counseling and health services on college and university campuses. J Am Coll Heal. 2010;58:583-96.

41. Eisenberg D, Golberstein E, Gollust SE. Help-seeking and access to mental health care in a university student population. Med Care. 2007;45:594-601.

42. Kilmer JR, Cronce JM, Larimer ME. College student drinking research from the 1940s to the future: where we have been and where we are going. J Stud Alcohol Drugs Suppl. 2014;75 Suppl 17:2635. College student drinking research from the 1940s to the future: where we have been and where we are going.

43. McKee SA, Falba T, O'Malley SS, Sindelar J, O'Connor PG. Smoking status as a clinical indicator for alcohol misuse in US adults. Arch Intern Med. 2007;167:716-21.

44. Harrison EL, Desai RA, McKee SA. Nondaily smoking and alcohol use, hazardous drinking, and alcohol diagnoses among young adults: findings from the NESARC.

45. Wiers RW, Rinck M, Dictus M, van den Wildenberg E. Relatively strong automatic appetitive action-tendencies in male carriers of the OPRM1 G-allele. Genes Brain Behav. 2009;8:101-6.

46. Van den Wildenberg E, Wiers RM, Janssen RGJH, Lambrichs EH, Smeets HJM, Van Breukelen GJP. A functional polymorphism of the mu-opioid receptor gene (OPRM1) influences cue-induced craving for alcohol in male heavy drinkers. Alcohol Clin Exp Res. 2007;31:1-10.

47. D'Onofrio G, Degutis LC. Preventive care in the emergency department: Screening and brief intervention for alcohol problems in the emergency department: a systematic review. Acad Emerg Med. 2002;9(6):627-38. 